\title{
Index
}

ABC (Abstain, Be faithful or wear a Condom) 80, 118

abortion 2-3, 8-9, 13, 16, 19, 21-3, 26, 29-31, 35, 40, 42, 57, 59-61, 66-9, 92, 104-5, 109, 112, 117-18, 120, 124-6, 150, 182, 193-225, 234-5, 237-8, 242-5 Abortion Non-Discrimination Act 217 'abstinence-only' 3, 105, 110-11 'abstinence-plus' 104, 110-11, 120, 127, 237

Administration for Children and Families (ACF) 110, 179-81 Adolescent Family Life Act (AFLA) 107, 109-10, 112

Adoption Promotion Act 215

AIDS 16, 33, 38, 67-8, 74, 77-80, 103, 105, 107, 112-13, 118-20 Alabama 81, 88, 107, 119-120, 138, 144, 239

Alan Guttmacher Institute 61, 107, 120, 205, 208, 225

Alito, Samuel 224, 236-7

Alliance for Marriage 85, 89

American Decency Association 144, 152, 159

American Enterprise Institute 6, 69 Arizona 75, 139, 182, 188

Arkansas 182, 188

Arkes, Hadley 85, 207, 214-15

Arlington Group 85-6, 95

Armey, Dick 57
Ashcroft, John 74, 78-9, 137, 141-2, 144, 148-52, 213

Ashcroft v. The Free Speech Coalition (2002) 150

Baehr v. Miike (1993) 41

Bauer, Gary 59, 85, 203

Bennett, William 15, 58, 62, 85, 140, 180, 207

Beverly LaHaye Institute 144

Blankenhorn, David 177, 182, 184-6

bloggers 26, 163

Bob and Carol and Ted and Alice 14

Bono 161

Bork, Robert 20, 242

Born-Alive Infants' Protection Act 214-15

Bowers v. Hardwick (1986) 81

Boy Scouts of America v. Dale (2000) 76

Bozell III, L. Brent 159

Brownback, Sam 56-7, 88, 115, 156, 182, 217, 245

Bubba the Love Sponge 162, 164

Buchanan, Patrick J. ('Pat') 18-19, 58, 74, 159, 210

Buckley, Wlliam F. 159

California 4, 65, 95-6, 109, 124-5, 146-7, 153, 206, 216-17, 221

Cambria List 141

Carter, Jimmy 2 
Cato Institute 64, 117, 163, 188

Cheney, Dick 75, 116, 212, 234-5

Child Interstate Abortion

Notification Act 220

Child Online Protection Act 148-9

Child Pornography Prevention Act 150

Christian Coalition 16, 18, 24-5, $55,57-8,116,153,177,204$, 210, 239-40

City of Los Angeles v. Alameda Books (2002) 154

Clinton, Bill 1, 3-4, 19, 20-1, 26, $28,31,35,41,53-8,63,68,78$, $104,108,113,123,140-2,152$, $177,178,179,186,187,206-8$, $211,213,215,220,224,233$, 234, 244, 246

Clinton, Hillary 19, 244-5

cohabitation $35,107,180$

Colorado 18, 56, 78, 85, 88, 92

Colson, Charles 48, 57, 86, 222

Communications Decency Act 56, 149

‘compassionate conservatism' 2,53 , 55

Concerned Women for America (CWA) 2, 18, 26, 82-3, 85, 114-15, 118, 143-5, 152, 163, $166,181-2,213,239$

Congress 6-7, 26-30, 41, 54-8, $63-4,75,80,82,85,87,93,102$, 107, 114-15, 118-19, 144-5, $150,158,161-3,166,177-81$, 185, 195, 204, 208, 213-14, 236, 246

Connecticut 96, 156, 244

contraception 13, 62, 92, 102, $104-5,113-17,120-1,124-5$, 127, 150, 211, 218, 245

Contract with America 54, 64, 178

Contract with the American Family

57, 153, 204

covenant marriage 182, 185

culture wars 5, 29, 102
Deep Throat 138-9

Defense of Marriage Act (DOMA) $28,41,56,84,86-7,89,93-5$, 214

DeLay, Tom 56, 115, 156, 239

demoralization 60, 62

Denmark 90-1

Desperate Housewives 164

District of Columbia 103

Dobson, Ed 58

Dobson, James 3, 56, 85, 139, 222-4, 238

Doe v. Bolton (1973) 194

Dole, Bob 19, 65, 75, 203, 209, 211, 233, 235, 246

Dworkin, Andrea 139

Eisenstadt v. Baird (1972) 15

Elders, Joycelyn 104

equal protection 81, 195, 217

evangelicals 17, 22, 49, 60, 69, 233

Evertz, Scott 77-9

Exodus 89-90

\section{FACE 202}

Falwell, Jerry 16, 35, 67

Family Guy 164

Family Research Council 2, 18, 26, $50,59,85,117,121,142,164$, $167,181,203,220,222-4$, 239-40, 246

Farris, Michael 77, 239

Fatal Attraction 35

FCC v. Pacifica Foundation (1978) 157

Federal Communications Commission (FCC) 157-66

Federal Marriage Amendment (FMA) 86-95, 235, 237-8

filibuster 137, 221, 223

First Things 57, 207

Florida 6, 47, 63, 66, 78, 81, 189, 219, 236

Flynt, Larry 141, 242 
Focus on the Family 2-3, 56, 85, 138-9, 144, 181, 222, 238

Food and Drug Administration (FDA) 113-15, 212-14

Fox News 26

Freedom of Access to Clinic Entrances 202

Frist, Bill 88, 93, 167, 218, 222-3, 239-40, 246

Frum, David 4

Galston, William 234

Georgia 49, 150

gerrymandering 7

Gingrich, Newt 28, 53-5, 187

Giuliani, Rudolph 155, 188

Goeglein, Tim 50, 92

Gonzales, Alberto 144, 151, 210, 238

Goodridge v. Department of Public Health (2003) 81, 84-8, 96

Gore, Al 41, 186, 244

Graham, Billy 48, 52

Greenberg, Stanley 3, 42

Griswold v. Connecticut (1965) 15, 150

gubernatorial conservatism 63

Hastert 55, 93

'healthy marriage' 69, 176, 179-82, 189-90

Heritage Foundation 105-6, 110, 123, 189, 241

heteronormativity 177

Himmelfarb, Gertrude 60

Holland 93

homosexuality $8,14,18,23,35,42$, 51, 66-7, 83, 89-90, 93, 97, 120, 126,182

Hormel, James 74, 77

Horn, Wade F. 110, 182, 185-7, 189

Horowitz, David 63, 163

House of Representatives 7, 17, 27, 88, 92, 113, 164, 178, 195, 217, 220, 238
Houston Gay and Lesbian Political Caucus 74

Hudson Institute 90, 182

Illinois $115-16$

indecency 156, 159, 161

Indiana 50, 92, 145

Institute for American Values 177, 182-3, 186

Islam 52

Jackson, Janet 162-3, 237

Johnson, Boris 65

Johnson, Lyndon B. 14

Kansas 18, 20, 56, 81, 88, 95, 156, 217, 245

Kennedy, Anthony 81, 150, 222, 239

Kennedy, John F. 145

Kentucky 88, 103, 124, 139, 222

Kerry, John F. 4, 116, 194, 234-5

Kinsey, Alfred 89

Kolbe, Jim 75, 80

Koop, C. Everett 67, 103

Kristol, William (Bill) 212, 224

Kristol, Irving 188

Kurtz, Stanley 90-3

Laci and Conner's Law see Unborn Victims of Violence Act

Lawrence v. Texas (2003) 76, 81-4, 150-1, 193

Lewinsky, Monica 26, 41, 55, 58, 244

Lieberman, Joseph 41, 140, 156, 244-5

Limbaugh, Rush 55

Log Cabin Republicans 74-6, 78-80

Lott, Trent 26

Louisiana 81, 94, 126, 182, 188-9, 220

McCain, John 5, 49, 75-7, 166, 211-12, 223 
Magnet, Myron 53

Maine 147, 217

Marriage Preparation and

Preservation Act 189

Marriage Protection Act 94

'marriage tax penalty' 176-8

Maryland 103

Massachusetts 15, 27, 66, 84-5, 87-8, 90, 92, 96, 115, 207, 234

Meese Commission 33, 139, 143, 155

Michigan 63, 66, 139

Miers, Harriet 223-4, 236-9

Miller 146-7, 153, 157, 241

Minnesota 88, 120, 187, 221

Missouri 50, 81, 94, 137, 205-6, 219

Murray, Charles 62, 139, 183

Musgrave amendment 85

Mychal Judge Police and Fire

Chaplains Public Safety Officers' Benefit Act 79

NARAL Pro-Choice America

(formerly the National Abortion Rights Action League) 197, 217

National Gay and Lesbian Task

Force 182

National Organization for Women

13, 187, 217, 221

National Review 85, 90, 92, 201, 214, 243

National Right to Life Committee (NRTLC) 195

National Sanctity of Life Day 219

Neuhaus, Richard J. 57, 207

New Hampshire 5, 18, 211-12

New Jersey 63, 66, 103

New Mexico 87

New York 4, 49, 63, 66, 87, 96, 115, 147, 155-7, 188, 197, 200-1, 208, 216

Nixon, Richard M. 13, 20, 22, 48, 52, 86, 222, 234

Norquist, Grover 59, 80, 240-1
North Carolina 81, 88, 158, 204

'nuclear option' 222-3, 239

O'Connor, Sandra Day 20, 81, 206, 223, 236

obscenity 14, 137-8, 140-3, 156

Ohio 3, 6, 63, 123, 235

Oklahoma 81, 88, 92, 110

Olasky, Marvin 53, 55

Oregon 87

Parents Television Council (PTC) 158-61, 163, 165-6

partial-birth abortion 216

partisanship 23

Pennsylvania 56, 83, 92, 112, 127, 139, 150, 156, 205, 208, 212, 219, 245

Perkins, Tony 85, 182, 223, 240

Personal Responsibility and Work Opportunity Act (PRWOA) 54, 56, 108-12, 119, 179, 186, 205

Pew Research Center 7-8, 89, 234

Philadelphia 38, 74, 149

Phillips, Kevin 47

Plan B 114-15, 117

Planned Parenthood 205, 210, 217, 220, 224

Planned Parenthood of Southeastern Pennsylvania (1992) 205

Ponnuru, Ramesh 201, 214, 225, 243

pornography 14, 19, 31-3, 35, $56,69,137-56,165,241-2$, 244

Powell, Michael 162-3, 203

premarital sex 14, 35

primaries 24-5, 49, 75, 111, 246

Promise Keepers 18

'purists' 9, 25, 201

Quayle Dan 41, 75

Reagan, Ronald 2, 17, 20, 22, 51, 54, 58, 64, 66-7, 105, 140, 142, 
184, 203, 205-6, 213, 219, 224, 234, 236-7, 246

Rector, Robert 105-6, 123, 189

Reed, Ralph 17, 24, 55, 58-9, 239-40, 246

Rehnquist, William 20, 148, 150, 223, 236

remoralization $60,62-3$

Reno, Janet 140, 142, 145, 149

Reno v. American Civil Liberties

Union (1997) 149

Republican National Coalition for Life 203, 208

Republican Study Committee 56

Republican Unity Coalition 80

'responsible fatherhood' 183

Rhode Island 217

Rice, Condoleezza 5

Roberts, John 223

Robertson, Pat 17, 49, 59, 116, 204, 222

Robinson, Gene 5

Roe v. Wade (1973) 15, 20, 150,

194, 203, 213-14, 217, 237

Roman Catholics 8, 18, 22, 190, 234

Romer v. Evans (1996) 78

Romney, Mitt 85, 115

Rove, Karl 1-3, 68-9, 80, 88, 224, 239

RU-486 208, 212-13, 220

Rust v. Sullivan (1991) 220

Santorum, Rick 83, 92, 115-16, 182, 214, 245

Scalia, Antonin 20, 81-3, 148, 213, 223, 237

Scandinavia 91, 93

Schlafly, Phyllis 21, 178, 203, 222, 239

Schwarzenegger, Arnold 95-6

'security moms' 234

Senate $6,17,20-1,26-7,57,74$, 84, 87-8, 93-5, 113, 127, 137-8, 144-5, 164-7, 181, 185, 187,
195, 203, 206-7, 217-18, 220-4, 238-40, 242, 244, 246

Sex and the City 164

sexual revolution 13-14, 16, 43, 102

Sexually-transmitted diseases (STDs) 100, 102-3, 106-7, 113, 116, 120

Shalala, Donna 104, 123

Sheldon, Lou 79

SIECUS (the Sexuality Information and Education Council of the United States) 102, 125, 128

South Carolina 49, 76-7, 81, 211

Southern Baptist Convention 33, 51

SPRANS (Special Projects of

Regional and National

Significance) 109-10, 112, 125

State Children's Health Insurance Program (SCHIP) 219

Stenberg v. Carhart (2000) 212, 221

Stern, Howard 158-9, 162, 164, 242

Stonewall 13

Supreme Court 15, 17, 19-20, 42, $76,78,81,83,144,146,148-50$, 153-4, 157, 166, 193, 204-6, 210, 212, 216, 220-5, 236-7, 242-3

Temporary Assistance to Needy Families (TANF) 54, 179-81, 189

Texas 7-8, 25, 47, 49-50, 63-6, 68, 74-6, 81-2, 88, 92, 110-11, $113,121,124,147,150,156$, 189, 209-10, 221, 223, 236

The Silver Ring Thing 18, 122

Thomas, Clarence 13, 17, 20, 58, 147-8, 223

Traditional Values Coalition 18, 79, 137, 152, 240

True Love Waits 18, 111, 122, 124

Uganda 80, 118

Unborn Victims of Violence Act

(UVVA) 216, 224 
United States v. American Library Association (2003) 146

US Conference of Catholic Bishops 218

values $4,16,19,21,29,41,49,76$, 107, 127, 177, 184, 188, 195, 233-5, 238, 243-4, 246

Values Action Team (VAT) 56-7, 144, 156

V-chip 160, 244

Vermont 95, 218

Virginia $24-5,81$
Washington Post 1, 8, 68, 95, 158, 224, 236, 239, 246

Washington Times 123, 142

Webster v. Reproductive Health Services (1989) 205-6

Weekly Standard 90, 92, 207, 212, 224

Weyrich, Paul 58, 80, 85

White House Office of Faith-Based and Community Initiatives 182

Wildmon, Donald 143, 152, 239

Wisconsin 63, 77, 206 